\title{
Optical fibre bending sensor for vehicle weight detection
}

\author{
Jan Jargus $^{1}$, Stanislav Kepak ${ }^{1}$, Jakub Cubik ${ }^{1}$, David Bujdos ${ }^{2}$, Martin Stolarik ${ }^{3}$ \\ ${ }^{1}$ Department of Telecommunications, VSB-Technical University of Ostrava \\ 17. listopadu 2172/15,708 00 Ostrava - Poruba, Czech republic \\ jan.jargus@vsb.cz; stanislav.kepak@vsb.cz; jakub.cubik@vsb.cz \\ ${ }^{2}$ Department of Building Materials and Diagnostics of Structures, VSB-Technical University of Ostrava \\ 17. listopadu 2172/15,708 00 Ostrava - Poruba, Czech republic \\ david.bujdos@vsb.cz \\ ${ }^{3}$ Department of Geotechnics and Underground Engineering, VSB-Technical University of Ostrava \\ 17. listopadu 2172/15,708 00 Ostrava - Poruba, Czech republic \\ martin.stolarik@vsb.cz
}

\begin{abstract}
This article describes vehicle detection sensor based on optical fibre bending. Initial measurements were performed on a Heckert FP 10/1 press. Based on these measurements, a prototype of a bending sensor for vehicle detection was designed. This prototype is intended primarily for static vehicle detection, where the magnitude of attenuation is proportional to the weight of the car. When measuring on this prototype, we tested 4 types of ethylene propylene diene methylene (EPDM) grooved rubber that pressed on an optical fibre located on smooth EPDM rubber base. Based on the measurements, we chose the EPDM rubber cover for which the detector had the greatest sensitivity. These initial results indicate that this can be a life-capable structure with a possible extension to a static weight sensor and, partly, to a WIM (weigh in motion) dynamic weight sensor.
\end{abstract}

Keywords: Optical fibre, Bending, Vehicle detection, Weight sensor, Weigh in motion.

\section{Introduction}

Currently there is a growing demand for road safety. This is related both to the detection and verification of vehicle weight limits and also to the ability to detect vehicles at critical points of the road, e.g. at railroad crossings. At the same time, efforts are made to detect vehicles and weight them at the same time, during regular traffic. This is the concept of Weigh-In-Motion (WIM), which is described in detail for example in [1-2]. There are many types of vehicle weight sensors based on different principles. They include piezoelectric sensors, sensors with mechanical elastic elements, FBG-based sensors, and optical fibre bending sensors. The piezoelectric sensors are discussed in [3-5]. The use of some special elastic elements for WIM is mentioned, for example, in [6-7]. The FBG principle finds use in many types of sensors and is also used for WIM applications [8-10]. Optical fibre bending sensors can be used for static and dynamic weight measurements. The special optical fibre used for WIM is described in [11] and some examples of fibre optic bending for static load measurements are in [12-14]. In this paper we present an initial measurement with a vehicle detector and weight sensor based on the bending of a common single-mode (SM) optical fibre.

\section{Operating principles}

Bends with a radius of less than $50 \mathrm{~mm}$ result in a greater degree of distortion of geometric conditions of the total reflection in the optical fibre, thereby losing the optical energy originally coupled to the core of the optical fibre. This loss is the greater, the smaller the bending radius is. The bending with a very small radius is called a microbending. The topic of optic fiber bending and its use for sensory purposes is discussed in many publications, e.g. in [15-17]. Microbending is done by the process of the spatial changes in the structure of an optical fiber. This stimulates couplings between the modes of optical fibre. The light power is coupled between the modes of fibre with propagation constants $\beta_{p}$ and $\beta_{q}$ satisfying following relation. 


$$
\beta_{p}-\beta_{q}=\frac{2 \pi}{\Lambda}
$$

where $\Lambda$ is the spatial frequency of micro bends. The correlation length for the wave coupling between the two modes should be approximately in the wavelength to the difference between the core and cladding refractive indices. The spatial period for a step increase of sensitivity to the microbending can be determined using the formula:

$$
\Lambda_{m}=\frac{\pi \cdot a}{\sqrt{\Delta}}=\frac{\sqrt{2} \pi a n_{0}}{N A}
$$

where $a$ is the radius of the optical fibre core, $n_{0}$ is the core refractive index for the working wavelength (e.g. $1550 \mathrm{~nm}$ ), and $N A$ is the numerical aperture of the fibre. For standard single mode telecommunications fibres and its working wavelengths this period is usually in the millimetres. This led to the following structural dimensions of the bending transducer used in the sensor. A detailed description of microbending losses is presented in [18-19], where the effect of the number of microbending and their period is analysed, as well as used wavelengths of light to the total loss of optical energy caused by bending.

\section{Experiments}

As a part of the experimental section, we first constructed the initial bending sensor, which we tested in the laboratories of the Faculty of Civil Engineering at the Technical University of Ostrava (TUO) on the Heckert FP 10/1 press. Based on the result of this measurement, we have developed a detection bending sensor for vehicles in several variants.

\subsection{Laboratory experiment}

Single-mode optical fibre of a total length of $3 \mathrm{~m}$ was inserted between an ethylene propylene diene methylene (EPDM) board and styrene-butadiene rubber (SBR) rubber plate of dimensions 40x60 cm, the lower EPDM board having smooth surface and the upper rubber having fine grooving. The fibre was attached to the lower EPDM board at several locations with a common adhesive tape and the fibre direction was perpendicular to the direction of the grooving of the upper rubber. Two ends of the fibre, each about $30 \mathrm{~cm}$ long, protruded from the microbending sensor. A detailed drawing depicting the layout of the optical SM fibre on the EPDM board is shown in the following figure:

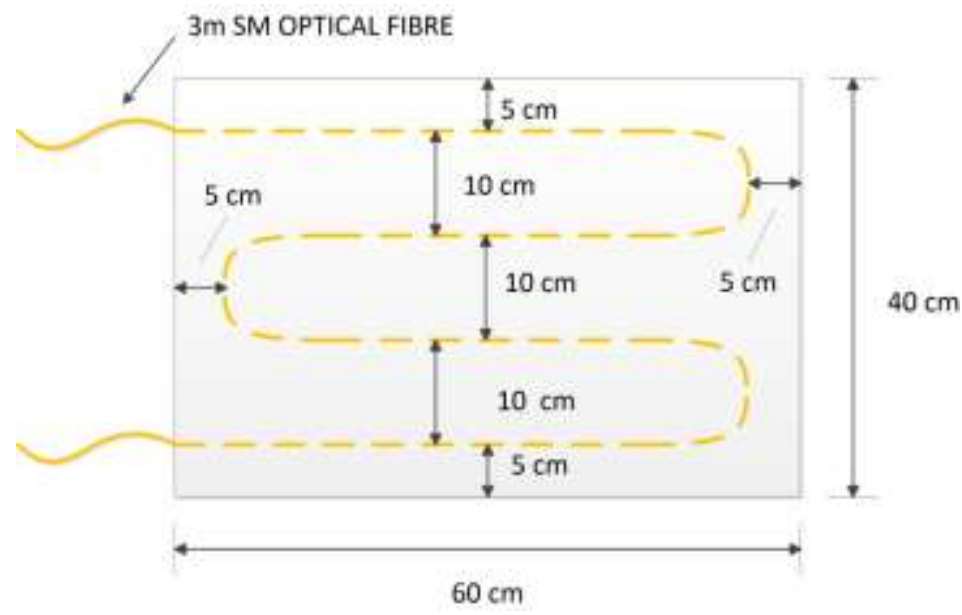

Fig. 1: The bottom part of the first bending sensor prototype. 
The lower part of the sensor consisted of an EPDM board of $5 \mathrm{~mm}$ thickness. The EPDM material has a hardness of $65^{\circ} \mathrm{ShA}$ and a strength of $3 \mathrm{MPa}$. The upper part of the sensor consisted of a $4 \mathrm{~mm}$ grooved rubber made of SBR rubber with a hardness of $60^{\circ} \mathrm{ShA}$ and a strength of $3 \mathrm{MPa}$. Figure 2 shows dimensions of the grooves of this rubber. The grooves were oriented perpendicular to the predominant direction of the optical fibre.

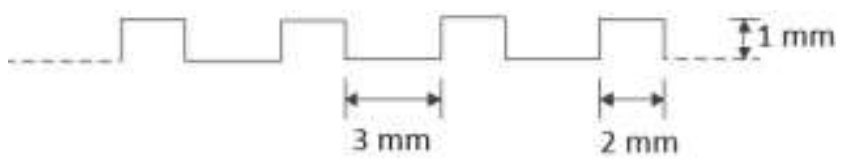

Fig. 2: The groove dimensions of the SBR rubber.

In the laboratory of the Faculty of Civil Engineering of the Technical University of Ostrava, we made an initial measurement with the proposed bending sensor. The bending sensor consisted of lower smooth EPDM plate, optical fibre, and top grooved SBR rubber. For measurement purposes, we placed this sensor on a metal plate under the press head of the press, the diameter of which was $16 \mathrm{~cm}$. The schematic diagram of the connection of the whole measuring assembly is shown in Figure 3 and with this measuring assembly, we performed three measurements at three different measuring points, as shown in Figure 4.

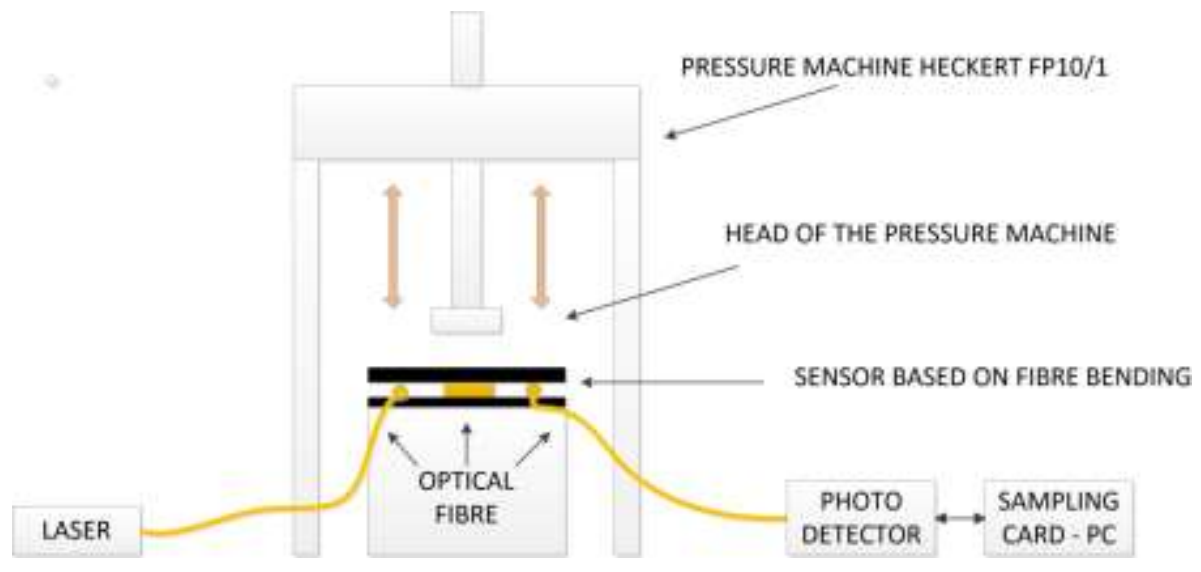

Fig. 3: The laboratory measuring assembly.

Figure 5 is a graph showing the time changes of the electrical voltage at the sampling card output for three different measuring points. These voltage changes were caused by gradual change in the compressive force that the press head had on the microbending sensor. The indicated time is rather indicative because the press used had manual adjustment and the rate of change of the compressive force of the press was re-set during the measurement according to the current change of electrical voltage. The laser wavelength used for the experiment was $1540 \mathrm{~nm}$.

During all measurements the pressure force increased from $0 \mathrm{~N}$ to the maximum of $10,000 \mathrm{~N}$, with longer duration of approximately $3,000 \mathrm{~N}$. We chose the longer duration for this value because the 3,000 N compressive force may roughly correspond to the compressive force that one wheel of a passenger car has on the road. The microbending sensor had the highest sensitivity for the measuring point 2. It seems that approximately half response at the measuring point 3 was caused by the fact that in this case the press head pressed only on one optical fibre, whereas in the case of measuring points 1 and 2, two fibres were compressed. To obtain an even response to the compressive force, we decided to redesign, assemble and test the microbending sensor with small lateral spacing of optical fibres. We promised that even more optical fibres would be uniformly loaded at almost any "measuring point", which would ensure the desired uniform response to the applied compressive force of a car tire. 


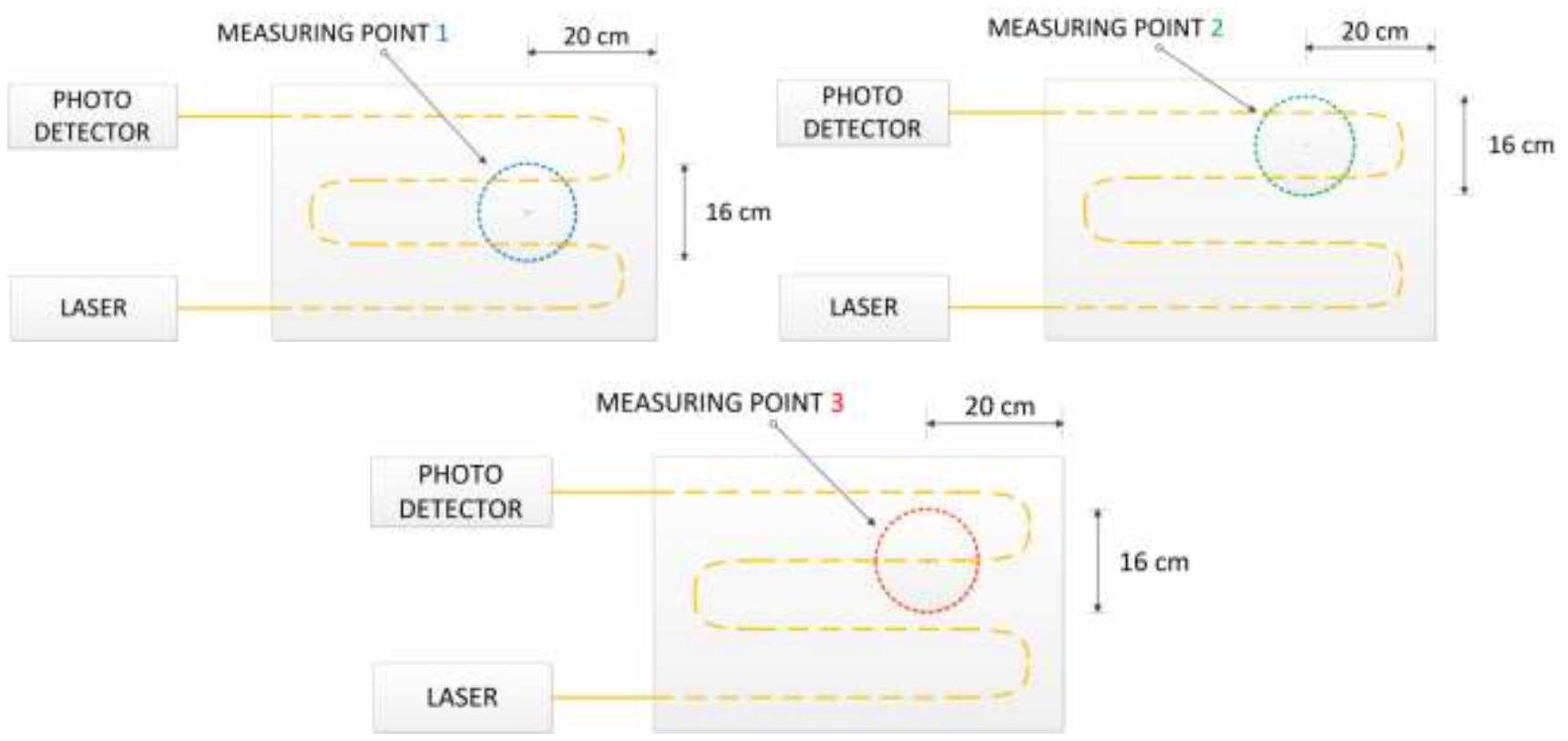

Fig. 4: Measurements points in the press machine.

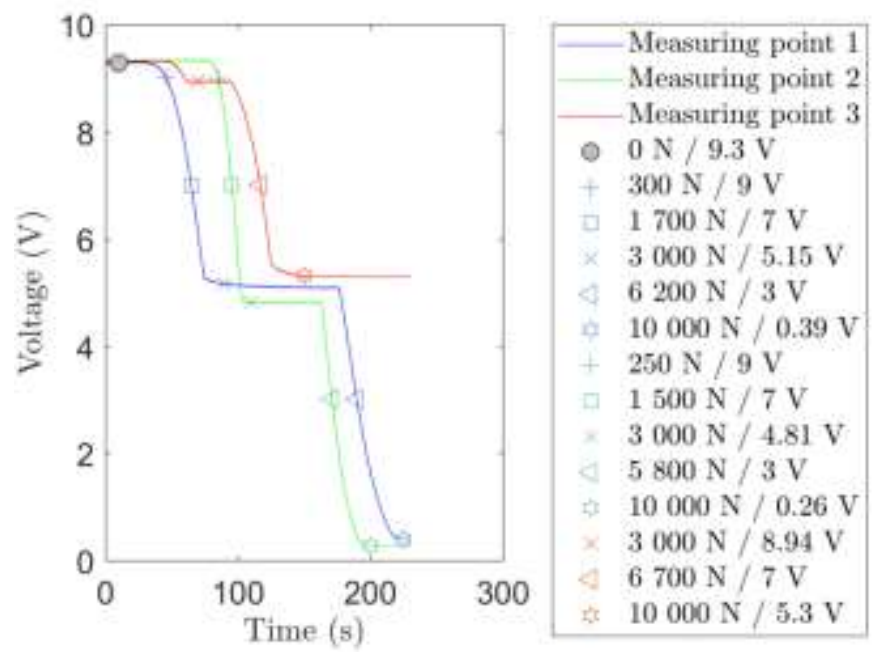

Fig. 5: The voltage changes caused by the change in the compressive force.

\subsection{Outdoor experiment}

A SM optical fibre of a total length of $50 \mathrm{~m}$ was attached to a smooth EPDM board of $250 \times 60 \mathrm{~cm}$. Due to the uniform sensitivity of the sensor the total of twenty lines of fibre was attached to this EPDM base board, see Figure 6 .

The lower part of the sensor consisted of $5 \mathrm{~mm}$ thick floor rubber made of EPDM rubber with a hardness of $65^{\circ} \mathrm{ShA}$ and a 3MPa strength. Four types of rubber were successively replaced on the upper part of the sensor, each rubber type being tested while laid in two $60 \mathrm{~cm}$ wide strips and $1 \mathrm{~m}$ long over the base plate in such a way as to allow the wheels of the car to slide on both stripes at the same time, see Figure 7.

These four types of rubber used as the upper part of the sensor were made of SBR rubber with a hardness of $60^{\circ} \mathrm{ShA}$ and a strength of $3 \mathrm{MPa}$ and had the following geometric parameters:

- Rubber Type 1: rough grooving - rubber thickness is $4 \mathrm{~mm}$

- Rubber Type 2: rough grooving - rubber thickness is $3 \mathrm{~mm}$ 
- Rubber Type 3: fine grooving - rubber thickness is $4 \mathrm{~mm}$

- Rubber Type 4: fine grooving - rubber thickness is $3 \mathrm{~mm}$

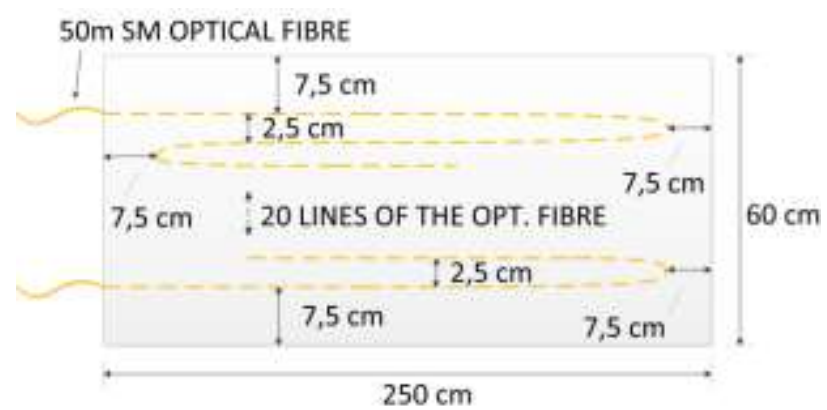

Fig. 6: The bottom part of the outdoor bending sensor.

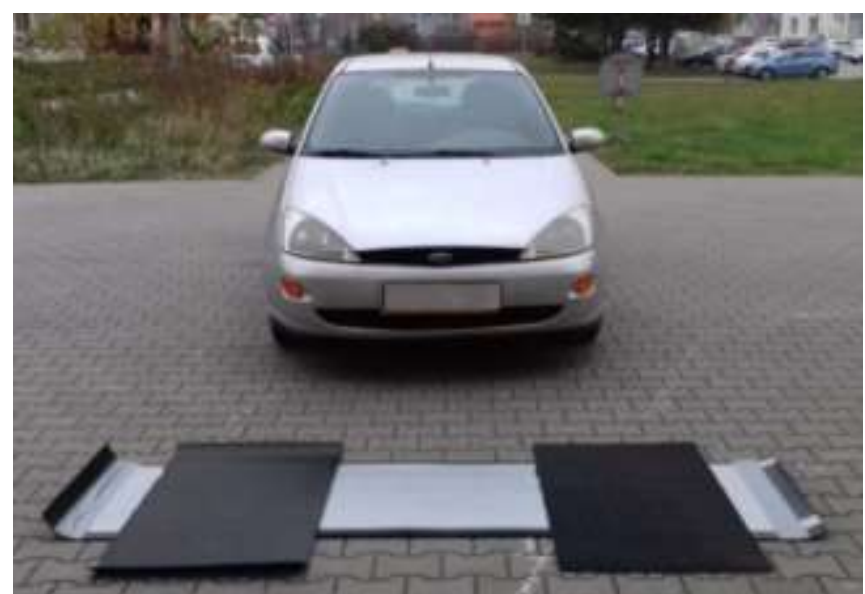

Fig. 7: The testing car and the outdoor bending sensor.

Figure 8 shows the geometric parameters of rubber types $1-4$.

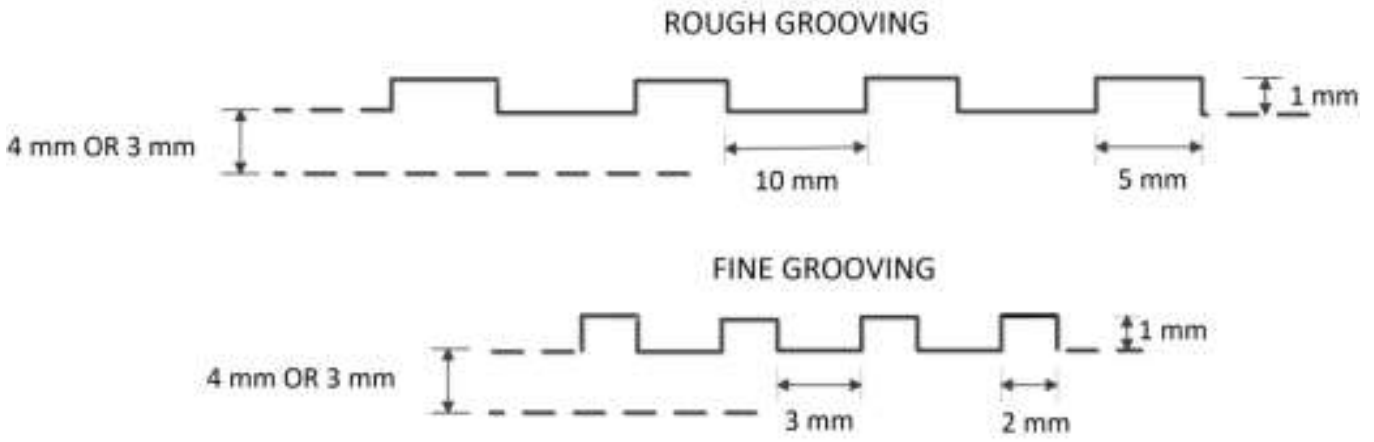

Fig. 8: The geometric parameters of the rubbers.

This outdoor measurement was carried out at the TUO campus. We connected the laser to one end of the fibre of this fibre optic sensor and photodetector and sampling card to the other end. As a top part of the sensor we used gradually all the types of the grooved rubber placed in two strips on the bottom of the bending sensor. These two top layers of rubber were slowly run over by a car, back and forth. Then we repeated this movement of the car for two more times, always with 
each axle lasting about 15 seconds on the tested rubber. For the testing, we used a Ford Focus car which carries a normal load of about $600 \mathrm{~kg}$ per axle. The course of the whole measurement was recorded on the sampling card and the results from the measurements are shown in Figure 9.
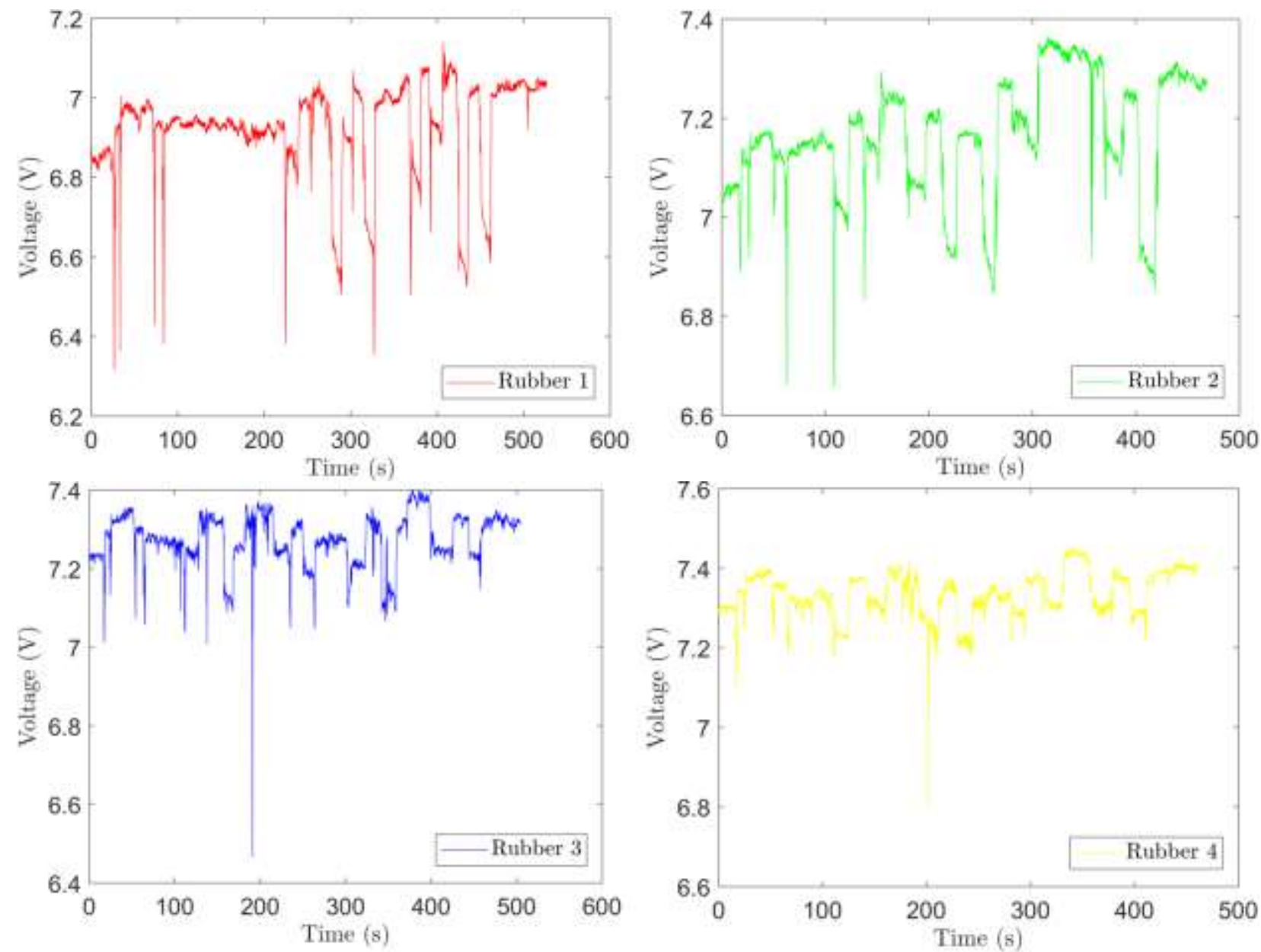

Fig. 9: The voltage changes caused by the car weight for rubber 1-4.

Table 1 summarizes the average change of electrical voltage on axle load for each type of rubber.

Table 1: Average changes in electrical voltage depending on sensor load with one axle of the car.

\begin{tabular}{|c|c|}
\hline Rubber Type & Average voltage response on an axle (V) \\
\hline Rubber 1 & $0.4-0.5$ \\
\hline Rubber 2 & $0.3-0.4$ \\
\hline Rubber 3 & $0.15-0.25$ \\
\hline Rubber 4 & 0.1 \\
\hline
\end{tabular}

\section{Results}

The graphs in Figure 9 shown the time changes of the electrical voltage at the output of the sampling card for the four different types of rubber that were used as the top of the microbending fibre optic sensor. These responses were caused by 
a change in the compressive force that the wheels of the car had on the bending sensor. It can be concluded from the graphs that the most sensitive was the type of rubber 1 , since it had the largest average change in voltage of about $0.5 \mathrm{~V}$. The smallest sensitivity was measured by the sensor for the type of rubber 4, since the average change in the voltage of about $0.1 \mathrm{~V}$. However, the whole measurement was distorted by how well the car wheels were driven to the top stripes of the tested types of rubber and how precisely they were able to stop on these stripes. For example, with the last type of rubber (with relatively low sensitivity), driver managed to precisely stop at the desired measurement points at the sensor, and for this reason, the corresponding graph of the change of the electrical voltage in dependence on car load is readable.

On each of the graphs we can first see four sharp dropping lines corresponding to the slow passage of the car though the sensor and each dropping corresponds to the passage of the car and to the dynamic load of the sensor by the vehicle's axle. It can be seen for instance on a graph in Figure 9 for the type of rubber 1 in the time range of about 0-100 sec. Then, there are always eight vehicle stops on the sensor, the stop time is approx. 15sec, the sensor response corresponds to the speed and position of the vehicle stop on the sensor, in Fig. 9 in a time of about 100 to 420 seconds.

\section{Conclusion}

The bending sensor had the highest sensitivity for rubber type 1 . The measurement was, however, influenced by the accuracy of running on the centres of the test rubbers and the duration of the stay on them. It can also be noted that the sensitivity of this sensor was not too high even though it was still sufficient. To increase sensitivity, it will be necessary to test other types of load plates of different hardness and with different grooving or different shapes of the deformation surface. Also a higher wavelength can be used to increase the sensitivity.

The overall concept of this bending sensor was primarily intended as a detection sensor for the weight detection of vehicles at critical points of the road, such as railway crossing. By detecting the presence of vehicles at these critical points, road safety can be significantly enhanced in the road section. The measurement results further indicate that after the optimization of components of this bending sensor there is a prerequisite for its use for indicative static vehicle weight measurement with possible dynamic load measurement for slower speeds of up to $50 \mathrm{~km} / \mathrm{h}$.

The results of this measurement were undoubtedly influenced by the ambient temperature. The experiment took place at a relatively cold temperature of about $5{ }^{\circ} \mathrm{C}$ when the hardness of the rubber used was increased. We will need to find out what results we could obtain at $30^{\circ} \mathrm{C}$ or in even colder environments $\left(-10^{\circ} \mathrm{C}\right)$. It would also be necessary to repeat the experiment several times to eliminate random events such as tire dimensions, the weight distribution of the vehicle, precision of the vehicle passage, etc. Optoelectronic parts of the system also had an impact on measurement accuracy, since the photodetector was not temperature stabilized. A change in ambient temperature could cause a change in the detected optical power, which is not a problem for dynamic measurements. On the contrary, the laser radiation source used in the experiment was thermally stabilized. In practice, a complex solution of optoelectronics with regard to temperature influences is expected.

\section{Acknowledgements}

This paper was supported by the Ministry of Industry and Trade of the Czech Republic project No. FV 10396 System for smart detection and collision conditions signalling for improving of railway security. The research described in this article could also be carried out thanks to the support of the projects No. SP2019/67 and SP2019/80. This research has been partially supported by the Ministry of Education, Youth and Sports of the Czech Republic through the grant project No. CZ.1.07/2.3.00/20.0217 within the frame of the operation program Education for Competitiveness financed by the European Structural Funds and from the state budget of the Czech Republic.

\section{References}

[1] C. E. Lee, "Standards for Highway Weigh-In-Motion (WIM) Systems," Standardization News, vol. 19, no. 2, pp. 3237, 1991.

[2] J. H. Wyman, "An Evaluation of Currently Available WIM Systems," in Proceedings of 3rd. National Conf. on Weigh-In-Motion, March 1989, pp. 6-176. 
[3] A. Kasahara and T. Fukuhara, "Measurement of the distribution of contact pressure of wide base tyres by means of a weigh-in-motion system," Heavy Vehicle Systems, vol. 3, no. 1-2, pp. 249-260, 1996.

[4] T. Pedersen and T. Haugen, "Vehicle weight estimation based on piezoelectric sensors used at traffic enforcement cameras experiences from the norwegian system," in Proceedings of the IEEE Conference on Intelligent Transportation Systems, Jokohama, Japan, 2018, vol. 2018-March, pp. 1-5.

[5] S. H. Vaziri, C. T. Haas, L. Rothenburg and R. C. Haas, "Investigation of the effect of weight factor on performance of piezoelectric weigh-in-motion sensors," Journal of Transportation Engineering, vol. 139, no. 9, pp. 913-922, 2013.

[6] R. Opitz, V. Goanta, P. Carlescu, P.-D. Barsanescu, N. Taranu and O. Banu, "Use of Finite Elements Analysis for a weigh-in-motion sensor design," Sensors, vol. 12, no. 6, pp. 6978-6994, 2012.

[7] T. D. Gillespie and S. M. Karamihas, "Feasibility of multiple-sensor weighing for increased accuracy of WIM," Heavy Vehicle Systems, vol. 3, no. 1-2, pp. 149-164, 1996.

[8] S.-Z. Chen, G. Wu, D.-C. Feng ad L. Zhang, "Development of a Bridge Weigh-in-Motion System Based on LongGauge Fiber Bragg Grating Sensors, "Journal of Bridge Engineering, vol. 23, no. 9, 2018.

[9] G. Kouroussis, D. Kinet, V. Moeyaert, J. Dupuy and C. Caucheteur, "Railway structure monitoring solutions using fibre Bragg grating sensors," International Journal of Rail Transportation, vol. 4, no. 3, pp. 135-150, 2016.

[10] Y. Huang, L. Palek, R. Strommen, B. Worel and G. Chen, "Real-time weigh-in-motion measurement using fiber Bragg grating sensors," in Proceedings of SPIE Sensors and Smart Structures Technologies for Civil, Mechanical, and Aerospace Systems, San Diego, CA, 2014, vol. 9061.

[11] R. B. Malla, A. Sen and N. W. Garrick, "A special fiber optic sensor for measuring wheel loads of vehicles on highways," Sensors, vol. 8, no. 4, pp. 2551-2568, 2008.

[12] R. K. Ula and D. U. Hanto, "Performance weight sensor using graded index optical fiber on static test with UTM," Journal of Physics: Conference Series, vol. 853, no. 1, 2017.

[13] J. Jargus, J. Nedoma, M. Fajkus, M. Novak, P. Mec, D. Cvejn, D. Bujdos and V. Vasinek, ”Analysis of using PDMS polymer as the sensors of the pressure or weight," in Proceedings of SPIE, Warsaw, Poland, 2017, vol. 10440.

[14] A. Vijayan, S. Gawli, A. Kulkarni, R. N. Karekar and R. C. Aiyer, "An optical fiber weighing sensor based on bending," Measurement Science and Technology, vol. 19, no. 10, 2008.

[15] Q. Wang, G. Farrell and T. Freir, "Theoretical and experimental investigations of macro-bend Losses for standard single mode fibers," Optics Express, vol. 13, no. 12, pp. 4476-4484, 2005.

[16] H. F. Taylor, "Bending Effects in Optical Fibers," Journal of Lightwave Technology, vol. 2, no. 5, pp. 617-628, 1984.

[17] S. Likoglu, K. Alemdar, K. Fidanboylu and O. Toker, "A novel microbending hetero-core fiber optic sensor for force and location sensing with applications to home security," in Proceedings of SPIE, San Diego, CA, 2014, vol. 9062.

[18] A. J. Harris and P. F. Castle, "Bend Loss Measurements on High Numerical Aperture Single-Mode Fibers as a Function of Wavelength and Bend Radius," Journal of Lightwave Technology, vol. 4, no. 1, pp. 34-40, 1986.

[19] V. Arya, K. A. Murphy, A. Wang and R. O. Claus, "Microbend Losses in Singlemode Optical Fibers: Theoretical and Experimental Investigation," Journal of Lightwave Technology, vol. 13, no. 10, pp. 1998-2002, 1995. 